\title{
Hypothalamic Ventromedial Nuclei Amplify Circadian Rhythms: Do They Contain a Food-Entrained Endogenous Oscillator?
}

\author{
SuJean Choi, Lai Sze Wong, Cherrilyn Yamat, and Mary F. Dallman \\ Department of Physiology, University of California San Francisco, San Francisco, California 94143-0444
}

Several endogenous oscillators determine circadian rhythms. One, light-entrained, is in the suprachiasmatic nuclei (SCN), the others, food-entrained, are in unknown sites. To determine how the hypothalamic ventromedial nuclei (VMN) and feeding affect rhythms, we compared nocturnally active rats fed either ad libitum or for $2 \mathrm{hr} / \mathrm{d}$ during light [restricted feeding (RF)] and either with or without colchicine-induced disruption of VMN. We measured rhythms in temperature, locomotor activity, feeding, drinking, corticosterone, and the numbers of cells expressing c-Fos in light/dark in hypothalamic nuclei, the suprachiasmatic nuclei, and two major SCN targets, the subparaventricular zone (sPVNz) and paraventricular thalamus (pvTHAL). c-Fos cells were always light $>$ dark in SCN, whereas the VMN and SPVNz lacked light/dark differences except after RF and RF plus VMN disruption, respectively. Controls fed ad libitum had highamplitude rhythms and, generally, c-Fos cells dark > light. In
RF controls, a c-Fos pattern dark > light occurred in VMN; generally, c-Fos cell numbers increased elsewhere maintaining dark > light. By contrast, levels of corticosterone peaked before food. In rats fed ad libitum, VMN with colchicine markedly reduced rhythm amplitudes, not phase. c-Fos patterns were abolished except in pvTHAL and SCN. In RF, VMN disruption blocked corticosterone and light/dark c-Fos patterns in all nuclei but produced a pattern in the SPVNz like SCN. We conclude that VMN amplify rhythmic output from the SCN, and the RF-induced rhythm in VMN enhances c-Fos activity driven by the SCN. The VMN may contain a food-entrained oscillator, and the sPVNz may integrate output from several oscillators.

Key words: hypothalamus; suprachiasmatic nucleus; ventromedial nucleus; circadian rhythms; endogenous oscillators; subparaventricular zone; c-Fos protein
Entrained by photic cues, endogenous circadian activity of the suprachiasmatic nuclei (SCN) is essential for expression of circadian rhythms in a wide range of behavioral and homeostatic systems (Stephan and Zucker, 1972; Turek, 1985). The SCN exhibit cyclic changes in c-Fos protein and mRNA expression with higher levels (Koibuchi et al., 1992) and increased electrical activity in vivo and in vitro during the light (Inouye and Kawamura, 1979; Green and Gillette, 1982; Meijer et al., 1997). Major SCN efferents target the hypothalamic subparaventricular zone $(\mathrm{sPVNz})$ and the paraventricular thalamus (pvTHAL) which project, in parallel with sparse direct SCN innervation, to ventromedial nuclei (VMN), dorsomedial nuclei (DMN), paraventricular nuclei (PVN), medial preoptic area (mPOA), and the arcuate nuclei (ARC) (Watts et al., 1987). These nuclei are important for regulation of energy balance (Dallman et al., 1993).

A second endogenous circadian oscillator, separate from the clock in SCN, is entrained by food intake (Mistlberger, 1994). Scheduled food and water restriction causes activity in the hypothalamic-pituitary-adrenal (HPA) axis, locomotor activity, and core temperature to peak around the time of food presentation

\footnotetext{
Received Dec. 23, 1997; revised Feb. 20, 1998; accepted Feb. 25, 1998.

This work was supported in part by grants from the American Diabetes Association and National Institute of Diabetes and Digestive and Kidney Diseases Grants DK-28172 and DK-09519 to S.C., National Center Research Resources Minority Initiative Grant R25RR10194 to L.S.W., and the American Chemical Society to C.Y. We thank Seema Bhatnagar and Alan Chu for their invaluable advice and assistance.

Parts of this paper have been presented in abstract form at the 1997 Annual Meeting of The Endocrine Society and 1997 Annual Meeting of the Society for Neuroscience.

Correspondence should be addressed to SuJean Choi, Department of Physiology, Box 0444, University of California San Francisco, San Francisco, CA 94143-0444. Copyright (C) 1998 Society for Neuroscience $\quad 0270-6474 / 98 / 183843-10 \$ 05.00 / 0$
}

(Bolles and Stokes, 1965; Mouret and Bobillier, 1971; Johnson and Levine, 1973; Krieger, 1974; Moberg et al., 1975; Gray et al., 1978; Wilkinson et al., 1979). Circadian rhythms emerge in rats with SCN lesions under conditions of restricted feeding (RF) and, after entrainment, can be shown to persist in the absence of food (Krieger et al., 1977; Honma et al., 1983a; Inouye, 1983; Stephan and Becker, 1989; Mistlberger, 1994).

Entrainment of HPA axis and anticipatory activity to RF depends on the amount of food intake (Honma et al., 1983a,b; Stephan and Becker, 1989); prolonged periods of access to food that allow normal intake and weight gain do not shift rhythms from the normal nocturnal peak. Thus, requirements for shifting rhythms appear to be both caloric restriction and food presentation out of phase with the light-entrained oscillator. RF rats reflect diminished caloric stores by mean elevation in HPA activity and decreased insulin, body weight, and fat weight compared with rats fed ad libitum (Wilkinson et al., 1979; Honma et al., 1983a; Persons et al., 1993). Entrainment of rhythms by restricted feeding requires activity in the $\mathrm{VMN}$, because short-term, although not long-term, lesions of the VMN (Krieger, 1980; Mistlberger and Rechtschaffen, 1984; Honma et al., 1987), but not the SCN (Krieger et al., 1977; Inouye, 1983), block rhythms induced by RF. RF allows analysis of interactions between the VMN and SCN-induced rhythms.

The neural pathways by which RF signals shifts in rhythms and the role, if any, of the VMN in this are untested. We have used c-Fos immunocytochemistry to examine neuronal activity (Sagar et al., 1988; Dragunow and Faull, 1989) in hypothalamic and extrahypothalamic sites at which the VMN and SCN would be expected to exert regulatory and/or oscillatory influences, based on anatomical and functional studies. ARC, PVN, DMN, para- 
ventricular thalamus, $\mathrm{SPVNz}$, and $\mathrm{mPOA}$ receive projections from the VMN and contribute to energy homeostasis (Luiten et al., 1987; Canteras et al., 1994). The ARC and PVN are known to regulate the HPA axis: feeding, drinking, and autonomic activity (Tokunaga et al., 1986; Touzani and Velley, 1992; Hanson and Dallman, 1995; Levin and Routh 1996), whereas DMN regulate locomotor activity (Lewis et al., 1993), and the mPOA is key to regulation of core temperature (Refinetti and Menaker, 1992). The pvTHAL may integrate external and internal stimuli and may receive projections from SCN and hypothalamic nuclei (Moga et al., 1995; Peng et al., 1995; Bhatnagar and Dallman, 1997).

Our experiments test the role of the VMN in rhythms in function and activation of brain sites. We examined rhythms in the HPA axis, core temperature, locomotor activity, and patterns of c-Fos protein expression after colchicine-induced disruption of $\mathrm{VMN}$ in rats fed ad libitum. We separated rhythms generated by the SCN from those regulated by feeding in RF rats and measured patterns in corticosterone and c-Fos protein in VMN with and without colchicine.

\section{MATERIALS AND METHODS}

In all experiments, we used male Sprague Dawley-derived rats weighing 200-240 gm (Bantin-Kingman, Fremont, CA) and housed them singly in a $12 \mathrm{hr}$ light/dark cycle (lights on at 7:00 A.M.). All animals were maintained on free access to Purina Rat Chow (2008) and water unless specifically stated. All experiments and procedures were approved by the University of California San Francisco Committee on Animal Research.

\section{Surgery and intracerebral injections}

All rats were placed in a stereotaxic apparatus after injections of a rodent anesthetic mixture consisting of ketamine, xylazine, and acepromazine $(77,1.5$, and $1.5 \mathrm{mg} / \mathrm{ml}$, respectively; $1 \mathrm{ml} / \mathrm{kg}$, i.p.). The scalp was incised in the midline and retracted, the skull was cleaned, and two small holes were drilled through it. After a 28 gauge needle connected to a microsyringe (Hamilton) was lowered to the appropriate coordinates, sterile saline $(0.1 \mu \mathrm{l})$ or colchicine $(1 \mu \mathrm{g} / 0.1 \mu \mathrm{l})$ [50:50 colchicine/fluorescein (Molecular Probes, Eugene, OR)/colchicine (Sigma, St. Louis, MO) mixture] in sterile saline was injected into the VMN bilaterally. With the upper incisor bar positioned at $-3.3 \mathrm{~mm}$ below horizontal zero, injections were made at the following coordinates: $2.6 \mathrm{~mm}$ posterior from bregma, $0.7 \mathrm{~mm}$ lateral to the midsaggital suture, and $9.2 \mathrm{~mm}$ below the surface of the skull (Paxinos and Watson, 1986). All intracerebral injections were made over $1 \mathrm{~min}$ followed by a $5 \mathrm{~min}$ period before the syringe was removed. The scalp was sewn, and the rats were allowed to recover from anesthesia before returning them to their cages in the animal room. On completion of each experiment, brains were post-fixed in $10 \%$ formalin for $24 \mathrm{hr}$ and then stored in a $30 \%$ sucrose in $0.1 \mathrm{M}$ PBS solution at $4^{\circ} \mathrm{C}$ or perfused and fixed with $0.15 \mathrm{M}$ phosphate buffer and ice-cold $4 \%$ formalin.

\section{Experiments}

Study 1. This study characterized daily rhythms in both core body temperature and locomotor activity after disruption of VMN function with colchicine. After calibration, biotelemetry temperature transmitters (model TM; Mini-Mitter Inc., Sunriver, OR) were inserted intraperitoneally under anesthesia and were monitored for $7 \mathrm{~d}$ to ensure a stable baseline of activity. Transmitted temperature and activity signals were picked up every 10 min by an antenna located outside the cage. Subsequently, telemetry data were averaged in $1 \mathrm{hr}$ bins. On day 7, sterile saline or colchicine was injected bilaterally into the VMN (seven rats per group). After surgery, core body temperature and locomotor activity were measured for $15 \mathrm{~d}$. Body weight and food consumption were measured on days $4-7$ with a $24 \mathrm{hr}$ fast occurring on day 5. A power outage caused $42 \mathrm{hr}$ of data to be lost.

Study 2a. We examined the circadian responses of behavioral and neuroendocrine systems to VMN disruption with colchicine. Food and water consumption were measured twice daily (at 10 and $14 \mathrm{hr}$ intervals during the light/dark period) for $2 \mathrm{~d}$ before and up to $5 \mathrm{~d}$ after surgery. Food consumption was calculated by the weight of noningested and spilled food at the end of the measurement period subtracted from the initial weight of the food placed into food bins. On day 5, in both salineand colchicine-injected animals (16 rats per group), blood samples were taken without anesthesia after lights on in the morning (9:00-10:30 A.M.) or just before lights off in the evening (6:00-7:00 P.M.) from a small incision made in a lateral tail vein (Akana et al., 1992). Blood $(\sim 0.2$ $\mathrm{ml}$ ) was collected in chilled tubes containing $0.3 \mathrm{M}$ disodium EDTA (10 $\mu \mathrm{l} /$ tube). Corticosterone (B) and insulin levels were measured in plasma from blood collected at 0 and $30 \mathrm{~min}$ after restraint. The basal blood collection was complete within $60 \mathrm{sec}$ of removing the rat from its cage and placing it in a Plexiglas restraining tube. A second blood sample was obtained after 30 min restraint (Akana et al., 1992). After the restraint period, rats were decapitated, and trunk blood $(5 \mathrm{ml})$ was collected in chilled tubes containing $0.3 \mathrm{M}$ disodium EDTA (100 $\mu \mathrm{l} /$ tube).

Study $2 b$. To characterize HPA activity after VMN with colchicine in greater detail, another set of rats was injected with saline or colchicine (eight rats per group). Food consumption was measured for $3 \mathrm{~d}$ after surgery to be certain of VMN inhibition. Saline- and colchicine-injected rats were equally divided and assigned to two groups. Each group consisted of four saline- and four colchicine-treated animals. At the start of the experiment, a blood sample taken from a tail vein nick was collected from each unanesthetized animal in one of the two groups. Two hours later, blood samples were similarly collected from the second group of rats. Subsequent samples were collected from both groups every $4 \mathrm{hr}$ staggered at $2 \mathrm{hr}$ intervals. This syncopated pattern of blood collection was repeated throughout a $24 \mathrm{hr}$ period. All blood samples during the dark period were collected under dim red light. In the final analysis, values from both groups were pooled to represent corticosterone levels at $4 \mathrm{hr}$ intervals.

Study $3 a$. The first phase of this experiment assessed c-Fos expression $5 \mathrm{~d}$ after saline or colchicine injections into the VMN in rats fed ad libitum. Five days after injections of saline $(n=10)$ or colchicine $(n=16)$ into the VMN, blood samples were collected from the tails of unanesthetized rats for measurement of basal levels of B. Half of each group was anesthetized and perfused immediately before the usual time of food presentation during the light cycle; the other half was anesthetized and perfused $3 \mathrm{hr}$ after lights off during the dark cycle on the same day. In samples collected during the dark, rats were anesthetized under dim red light; when asleep, their heads were wrapped loosely with foil before taking them out of the animal room for perfusion that was accomplished in dim light.

Study $3 b$. The experiment was repeated, but the animals were allowed food for only $2 \mathrm{hr}$ daily, provided $2 \mathrm{hr}$ after lights on (RF). A limited period of food availability during the light period (a time when rats do not normally eat) has been shown to shift rhythms of body temperature, HPA activity, and locomotor activity to peak at the onset of food presentation instead of at the onset of, or during, darkness (Krieger, 1980). Food intake and body weight were measured daily during the protocol. After $16 \mathrm{~d}$ of RF, rats were divided into two groups and injected on the 17th day with either saline or colchicine into the VMN as described above. RF was continued, and $5 \mathrm{~d}$ after surgery, animals in the light, prefood, and dark phases of the experiment were anesthetized and perfused (described above).

Sections of brain were cut on a freezing microtome at $30 \mu \mathrm{m}$, and every section through the VMN was collected and stored in cryoprotectant. Free-floating sections to be reacted with antibodies to Fos protein were incubated with 3\% normal goat serum in Tris-PBS (TPBS) and $0.3 \%$ Triton X-100 (Sigma) solution for $60 \mathrm{~min}$ at room temperature. After this, sections were incubated overnight at room temperature with a rabbit polyclonal antiserum against the N-terminal region of the Fos protein (1:10,000; Santa Cruz Biotechnology, Santa Cruz, CA) in TPBS preabsorbed with rat liver powder (Organon Teknika-Cappell, Malvern, PA) to reduce background staining. Sections were subsequently incubated with a biotinylated anti-rabbit IgG in goat (Vector Laboratories, Burlingame, CA) diluted 1:200 in the above TPBS mixture for $1 \mathrm{hr}$ at room temperature, washed, and then incubated with an avidin-biotin-peroxidase complex (Vector) for $1 \mathrm{hr}$ at room temperature. 3,3Diaminobenzidine tetrahydrochloride (Sigma) with $0.4 \% \mathrm{NH}_{4} \mathrm{Cl}, 20 \%$ $\beta$-D-glucose, and $1 \%$ nickel ammonium sulfate were used to produce a black chromagen.

Hypothalamic and thalamic cell groups were analyzed at the following anterior and posterior levels based on the brain atlas of Paxinos and Watson (1986): SCN (bregma, $-1.30 \mathrm{~mm}$ ), anterior sPVNz (bregma, $-1.80 \mathrm{~mm}$ ), posterior sPV Nz (bregma, $-2.30 \mathrm{~mm}$ ), VMN (bregma, $-2.56 \mathrm{~mm}$ ), DMN (bregma, $-3.14 \mathrm{~mm}$ ), parvocellular PVN (par- 

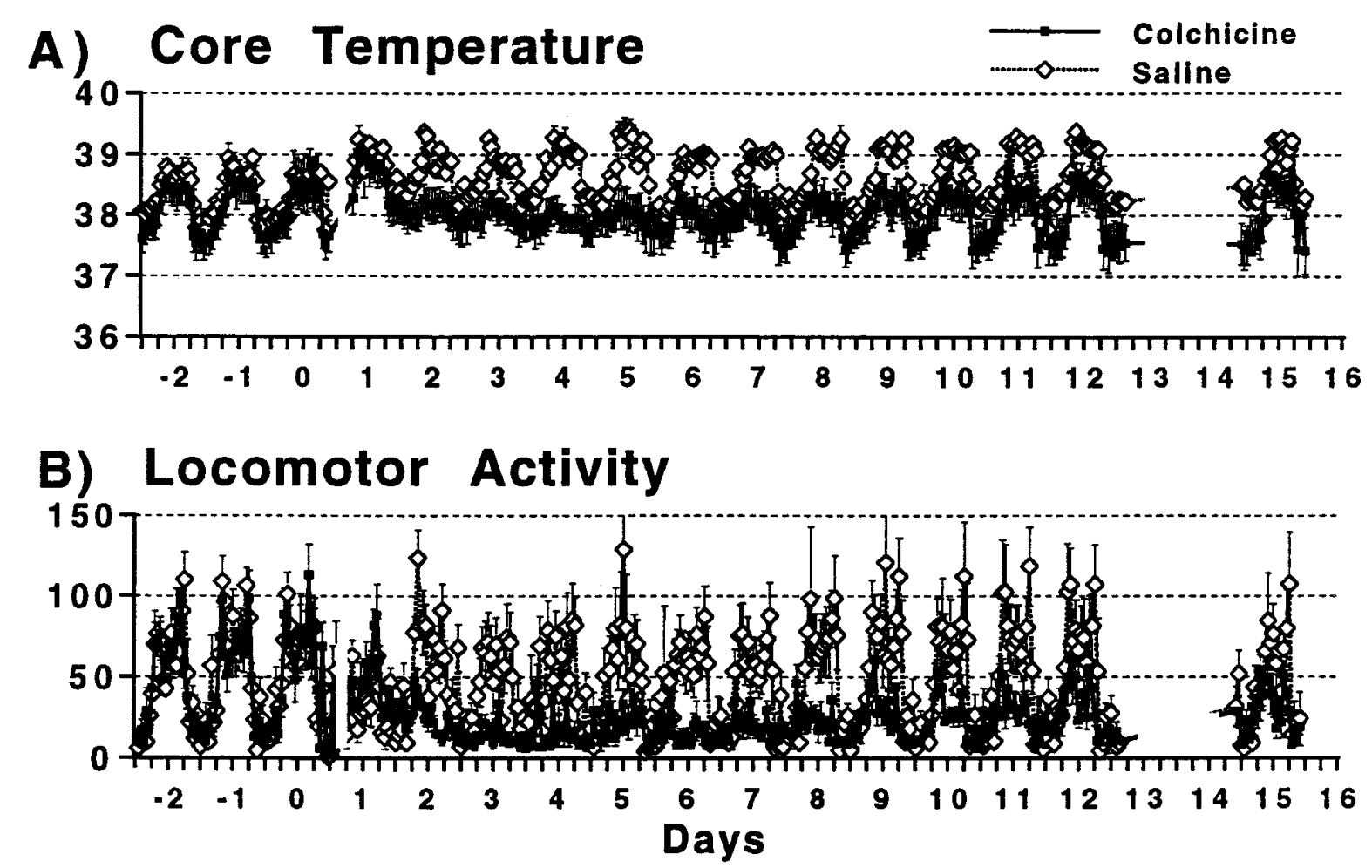

Figure 1. Core body temperature $(A)$ and locomotor activity $(B)$ in rats injected with saline $(n=6)$ or colchicine $(n=7)$ in the VMN. Colchicine injected into the VMN significantly diminished the amplitudes of both core temperature and locomotor activity. The gap at day 0 indicates the day of surgery, and the gap over days 13-14 was the result of a power outage.

voPVN) and magnocellular PVN (magnoPVN; bregma, $-1.80 \mathrm{~mm}$ ), mPOA (bregma, $-1.30 \mathrm{~mm}$ ), ARC (bregma, $-2.30 \mathrm{~mm}$ ), and pvTHAL (anterior bregma, -1.40 to $-1.80 \mathrm{~mm}$; medial bregma, -2.12 to -2.30 $\mathrm{mm}$; posterior bregma, -2.56 to $-2.80 \mathrm{~mm}$ ) (Bhatnagar and Dallman, 1997). We did not quantify c-Fos counts in any subdivision of these cell groups that has not already been specified. Throughout the analyses, we established particle size limits to distinguish c-Fos immunoreactive cells from staining of non-neuronal particles. Analysis of Fos-like immunoreactivity was measured using NIH Image software (W. Rasband, National Institutes of Health).

\section{Histological analysis}

In all animals with injections targeting the $\mathrm{VMN}$, histology was performed to locate the needle tracks and injection site in cresyl violetstained sections combined with adjacent fluorescent sections. Only rats with bilateral injections into the VMN and visible fluorescence contained within the VMN were included in the analyses and results. Analysis of the fluorescent spot confirmed our previous observation that at $5 \mathrm{~d}$ after injection the inactive fluorocolchicine (S. Choi and M. F. Dallman, unpublished observations) and presumably also the colchicine remained localized to the VMN (Choi et al., 1996). These criteria were achieved in $60-70 \%$ of the animals overall.

\section{Radioimmunoassays}

After centrifugation of the blood at $3000 \mathrm{rpm}$ and $4^{\circ} \mathrm{C}$, separated plasma was stored at $-20^{\circ} \mathrm{C}$ and used in radioimmunoassays for $\mathrm{B}$ and insulin. Plasma B was measured using an Immuchem double-antibody corticosterone RIA kit for rat (ICN Biochemicals, Costa Mesa, CA). The limit of detection was $1.5 \mathrm{ng}$ of $\mathrm{B} / \mathrm{ml}$. Plasma insulin was measured using a rat insulin RIA kit (Linco, St. Charles, MO). The limit of detection was $0.1 \mathrm{ng} / \mathrm{ml}$.

\section{Statistical analysis}

Data were analyzed using ANOVA corrected for repeated measures (when required). When main effects were significant, Scheffe analysis was used to test significance of post hoc effects. When only two groups were compared, Student's unpaired $t$ test was used. Telemetry data were analyzed by fitting a single cosine function to circadian data from each animal. The cosine function used was:

$$
y=A \times \cos (2 \pi t+\phi)+T
$$

where $y$ is the measured variable (temperature), $A$ is the amplitude, $t$ is the time of day, $\phi$ is the phase shift adjustment, and $T$ is the mean temperature.

All statistical analyses were conducted using commercial statistical software packages (StatView, Abacus Concepts, Calabasas, CA; DeltaGraph, Deltapoint Inc.). Statistical significance was established at $p \leq 0.05$. In all figures, mean data are accompanied by capped lines indicating SEM.

\section{RESULTS}

\section{Study 1}

Compared with saline-injected rats, colchicine-injected animals exhibited significant decreases in both mean temperature ( $p=$ 0.05 ; saline, $38.6 \pm 0.1$; colchicine, $38.0 \pm 0.3)$ and amplitude ( $p<0.0001$; saline, $0.48 \pm 0.02$; colchicine, $0.20 \pm 0.02)$ during days $1-7$ (Fig. 1). Presurgical mean temperature $(38.5 \pm 0.04)$ and amplitudes $(0.48 \pm 0.03)$ compared with postsurgical values (temperature, $38.6 \pm 0.1$; amplitude, $0.48 \pm 0.02)$ were not different in the saline-injected controls. Only amplitude significantly decreased after colchicine injections (postcolchicine, $0.2 \pm 0.02$ ) compared with presurgical values $(p<0.002$; precolchicine, $0.42 \pm 0.05$ ); mean temperature did not change (precolchicine, $38.0 \pm 0.3$; postcolchicine, $38.0 \pm 0.25$ ). During days $8-12$ there were no differences in mean temperature between colchicine- and saline-injected animals (colchicine, $38.0 \pm 0.3$; saline, $38.7 \pm$ 0.06). Although increasing in the colchicine-treated rats, the amplitudes of colchicine- and saline-treated rats were still signif- 


\section{Body Weight (g)}
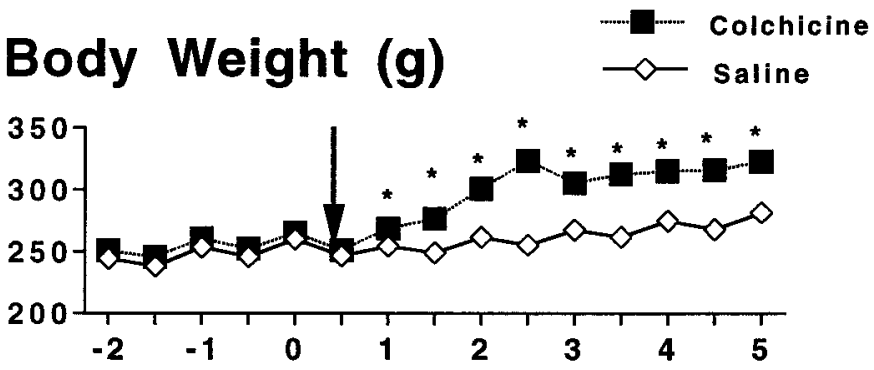

\section{Food Intake (g)}

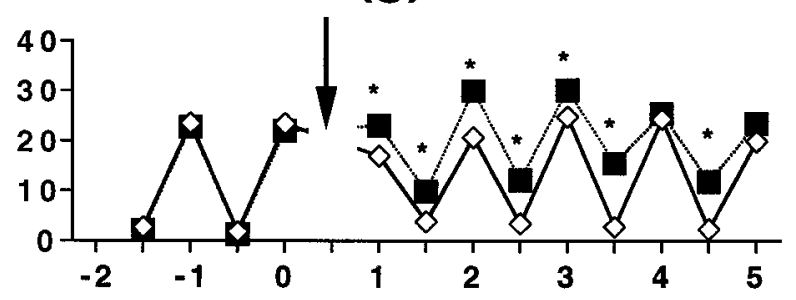

Water Intake (g)

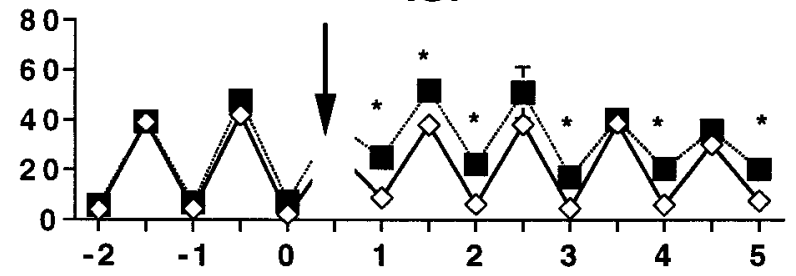

Figure 2. Changes in body weight (grams), food intake (grams), and water intake (grams) in rats injected with saline $(n=16)$ or colchicine $(n=9)$ in the VMN. Arrow, Surgery. *Significance $(p \leq 0.05)$.

icantly different during days 8-12 (colchicine, $0.45 \pm 0.02$; saline, $0.56 \pm 0.05)$ (Fig. 1A).

Both amplitude and quantity (mean) of locomotor activity were significantly decreased in colchicine-treated rats compared with saline-injected controls (amplitude, $25.2 \pm 4.0$; mean, $43.9 \pm 7.0$ ) $7 \mathrm{~d}$ after surgery. Although the amplitude increased significantly during days 8-12 compared with days $1-7$ after surgery $(p<$ 0.02 ), and the mean locomotor activity began to increase in colchicine-treated rats, both remained significantly different from controls ( $p<0.008$, and $p<0.04$, respectively) (Fig. $1 B$ ). Mean locomotor activity and amplitude of saline- and colchicinetreated rats were as follows, days 1-7: saline, mean, $43.9 \pm 7.0$, and amplitude, $25.2 \pm 4.0$; colchicine, mean, $21.3 \pm 2.0$, and amplitude, $5.4 \pm 0.8$; days $8-12$ : saline, mean, $49.8 \pm 11.0$, and amplitude, $25.8 \pm 2.0$; colchicine, mean, $38.1 \pm 7.0$, and amplitude, $14.5 \pm 3.0$. Only colchicine-treated rats exhibited significant decreases in mean locomotor activity and amplitude (days 1-7) compared with their own presurgical values (mean, $48.9 \pm 4.0$; amplitude, $32.5 \pm 4$; both $p<0.0001$ ).

Approximately 1 week after colchicine injections, the diurnal amplitudes of both body temperature and locomotor activity began to increase and return toward control levels. There was no effect of handling on days 4-7 or of an overnight fast on day 5 on either core temperature or locomotor activity.

\section{Study $2 a$}

Both body weight and food and water intake (Fig. 2) increased significantly in colchicine-injected rats compared with control

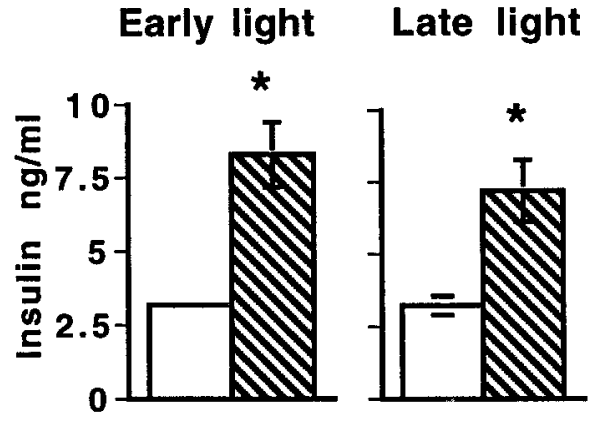

Figure 3. Insulin levels (nanograms per milliliter) in rats $5 \mathrm{~d}$ after they were injected with either saline (solid white bar) or colchicine (striped bar) in the VMN in both Early light (9:00 A.M.) and Late light (5:00 P.M.). Same rats as in Figure 2. ANOVA revealed a significant time of day effect $(p<0.0001)$. Post hoc, Early light, $p<0.0003$; Late light, $p<0.005$. Error bars indicate SEM. They are present, although some may be too small to be visible.

animals (ANOVA). There were significant effects of treatment, time, and a treatment $\times$ time interaction (all $p<0.0001)$. Three days after colchicine injections into the VMN, food intake in the light and dark periods were both significantly increased. However, by days $4-5$ food intake in light was still significantly elevated in rats injected in the VMN with colchicine, but food intake in dark decreased, thereby reducing the overall circadian amplitude compared with controls. The decreased amplitude of the light/dark period rhythm in water intake of colchicine-injected rats was more pronounced than that in food.

Insulin levels measured in colchicine-treated rats were higher than those in saline-injected controls in both the early and late light (Fig. 3). There was a significant time of day effect $(p<$ 0.0001 , ANOVA). Post hoc analysis confirmed a significant difference between saline- and colchicine-treated animals in both the early $(p<0.0003)$ and late $(p<0.005)$ light periods.

There were significant effects of time of day $(p<0.001)$ and interaction with treatment $(p<0.03)$ on basal B levels. Basal plasma $\mathrm{B}$ in the early light was significantly elevated $(p<0.04)$ and tended to be lower in the late light, thus reducing the amplitude of the daily rhythm (Fig. 4).

After 30 min restraint, there was a significant main effect of treatment on B levels $(p<0.0001)$ (Fig. 4). Colchicine-treated rats had decreased responses to restraint in both the early and late light periods ( $p<0.004$, and $p<0.002$, respectively).

\section{Study $\mathbf{2 b}$}

The basal excursion in plasma B over $24 \mathrm{hr}$ in colchicine-treated animals was markedly decreased in amplitude compared with controls (Fig. 5). This confirms the results of previous studies showing that morning basal B levels are elevated after VMNcolchicine. During the dark, basal B levels did not rise at the same rate or to the same extent as in controls, thus reducing the amplitude of response over the entire $24 \mathrm{hr}$ period. However, this damping effect occurred only in animals with anterior, not posterior, inhibition of the VMN.

\section{Study $\mathbf{3 b}$}

Restricted feeding was used to shift the peak of the rhythms to just before the period of restricted morning feeding, out of phase with the dark period. Body weight gain and food intake during RF were reduced (Fig. 6). After $7 \mathrm{~d}$ of food restriction, a modest increase in body weight and a plateau in food intake began to occur. Typically, colchicine-injected animals fed ad libitum show 

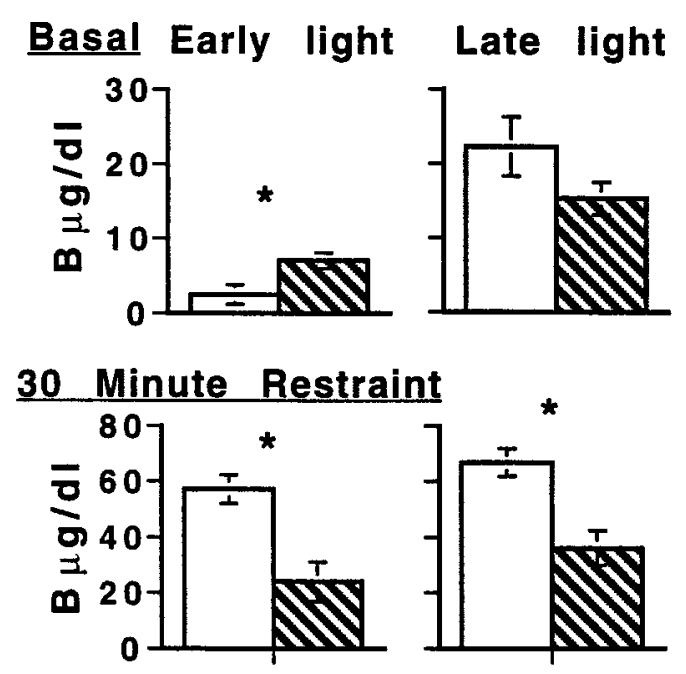

Figure 4. Diurnal basal and restraint-activated B in rats $5 \mathrm{~d}$ after injection of either saline (solid white bars) or colchicine (striped bars) in the VMN. Same rats as in Figure 2. There were significant effects of time of day $(p<0.0001)$ and interaction with treatment $(p<0.03)$ on basal B levels, whereas after restraint, B responses were significantly reduced by colchicine treatment $(p<0.0001)$. Early light, 9:00 A.M.; Late light, 5:00 P.M. *Significance $(p<0.04)$.

an immediate increase in both body weight and food intake compared with control rats (Fig. 2) (Choi et al., 1996). With the $\mathrm{RF}$ paradigm, colchicine injected into the VMN tended to decrease body weight and food intake compared with control animals, showing that these rats did not respond well to the challenge of restricted feeding. RF reversed the diurnal pattern in B from one that was significantly higher in the evening than in the morning $2 \mathrm{hr}$ after the onset of light $(p<0.0001)$ (Fig. 7, ad libitum) to a rhythm in which B was low in the evening ( $3 \mathrm{hr}$ after lights off) and high in the morning, before food ( $p<0.0001)$ (Fig. 7 , restricted food). After disruption of $\mathrm{VMN}$ function, there was no RF-entrained pattern in B (Fig. 7, restricted food).

\section{Studies $3 a$ and $3 \mathbf{b}$}

Ad libitum feeding

The SCN exhibited a pattern in c-Fos immunoreactivity with more protein-expressing cells in the light than dark (Fig. 8). By contrast, the number of c-Fos-immunostained cells was significantly higher in the dark than light in the DMN, parvoPVN (Fig. 9, $p P V N$ ) and magnoPVN (Fig. 10, $m P V N$ ) and in all regions of the pvTHAL (Fig. 11). The mPOA also tended to have increased numbers of c-Fos-immunostained cells in the dark $(p=0.057)$ (Fig. 10). In contrast, Figures 8-10 also show no light/dark differences in c-Fos expression in the sPVNz, the VMN, or the ARC.

Five days after bilateral colchicine injections into the VMN, the light/dark pattern in c-Fos protein expression remained in the SCN but was abolished in the DMN, parvoPVN, magnoPVN, medial preoptic area, and posterior pvTHAL (Figs. 8-11). Furthermore, colchicine injections into the VMN significantly increased c-Fos cell number in the VMN, DMN, and parvoPVN during the light.

\section{Restricted feeding}

Restricted feeding in control animals did not affect the phase of patterns in c-Fos-expressing cells in the SCN (Fig. 8), parvoPVN, magnoPVN, mPOA (Figs. 9, 10), or pvTHAL (Fig. 11). However,

\section{Anterior VMN}
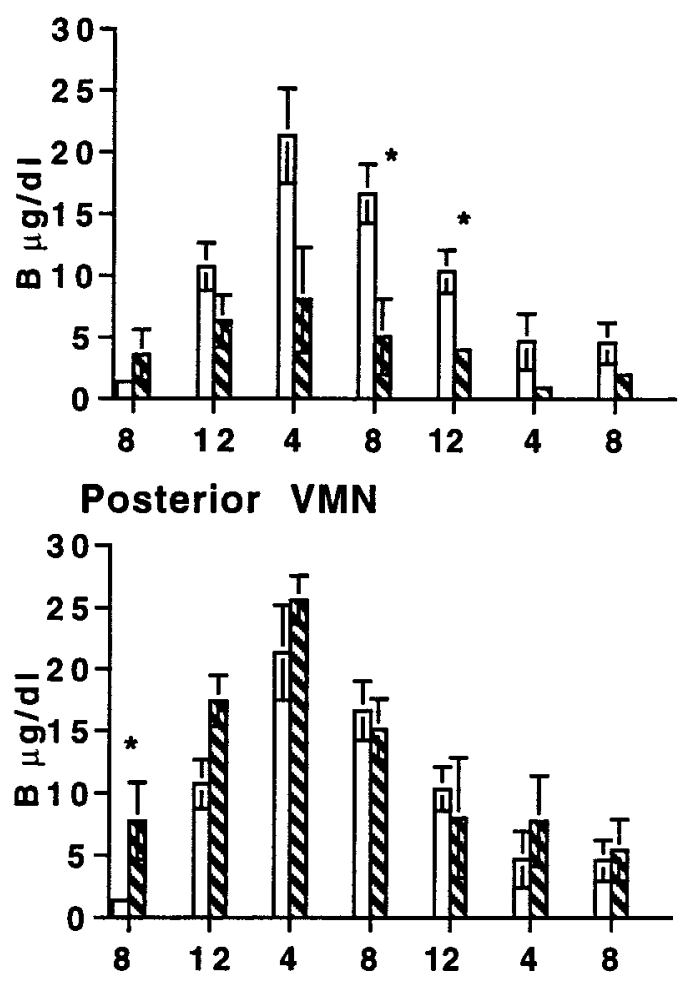

Figure 5. Basal plasma B during a 24 hr period (8:00-8:00 A.M.) 5 d after rats were injected with saline (solid white bars) or colchicine (striped bars) in the VMN. Top, Injections of colchicine in the anterior portion of the VMN (control, $n=4$; colchicine, $n=3$ ). Bottom, Injections of colchicine in the posterior portion of the VMN (control, $n=4$; colchicine, $n=3)$. *Significance $(p \leq 0.05)$.

RF did eliminate the pattern in the DMN (Fig. 9). Interestingly, in $\mathrm{RF}$ rats, there was a marked, overall increase in c-Fos-positive cells, and also a pattern emerged in the VMN of control animals with more c-Fos-positive cells in dark than light. This is like all other patterns except those in the SCN. A c-Fos pattern did not exist in the VMN of animals fed ad libitum (Fig. 9). As in animals fed ad libitum, the sPV Nz and ARC did not exhibit any light/dark differences in c-Fos expression (Figs. 8, 11).

Similar to the results in rats fed ad libitum, colchicine injections into the VMN of RF animals abolished patterns of c-Fos expression in the $\mathrm{VMN}$, parvoPVN, magnoPVN, mPOA, and the entire extent of the pvTHAL (Figs. 9-11). In marked contrast, the sPVNz did express a significant light/dark pattern after colchicine injections in RF animals similar to that in SCN (Fig. 8) $(p \leq 0.05)$.

\section{Missed VMN injections}

We have previously shown the lack of effect of injections of colchicine that missed the VMN on food intake and body weight gain (Choi et al., 1996). Furthermore, we have reported that we can clearly distinguish among the effects of bilateral injections of colchicine into the PVN, ARC vs DMN vs VMN (Choi et al., 1997), suggesting strongly that placement and discrete localization is important for the changes that occur after injections of colchicine into the VMN. In Study $3 b$ there were four rats with missed injections in the light group and three rats with missed injections in the dark group that were RF. In the VMN of the 


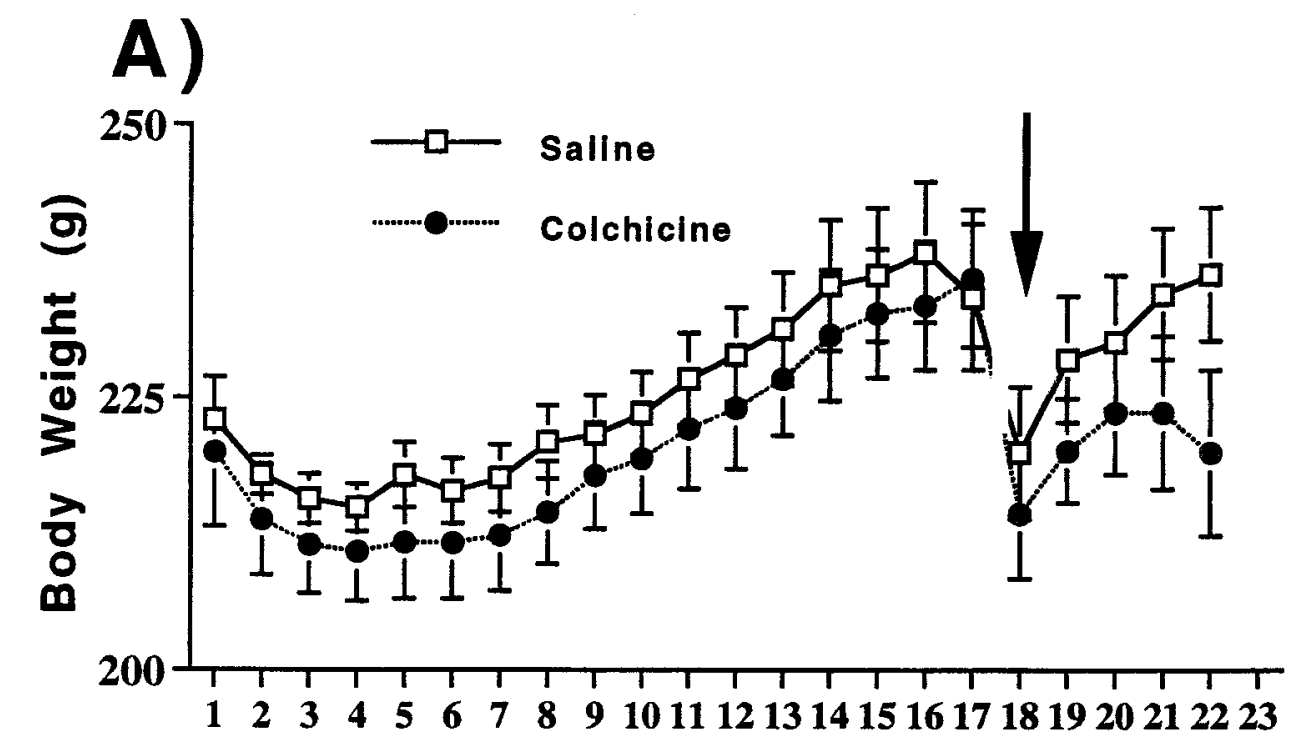

Figure 6. Body weight $(A)$ and food intake $(B)$ in rats fed for $2 \mathrm{hr}$ in the morning (RF) for $23 \mathrm{~d}$. Food intake occurred between 9:00 and 11:00 A.M. Arrow, Injections of either saline $(n=13)$ or colchicine $(n=10)$ into the VMN on day 17. Both body weight and food intake increased with time $(p<0.001)$. Although colchicinetreated rats tended to have decreased body weight and food intake, there were no differences between groups.

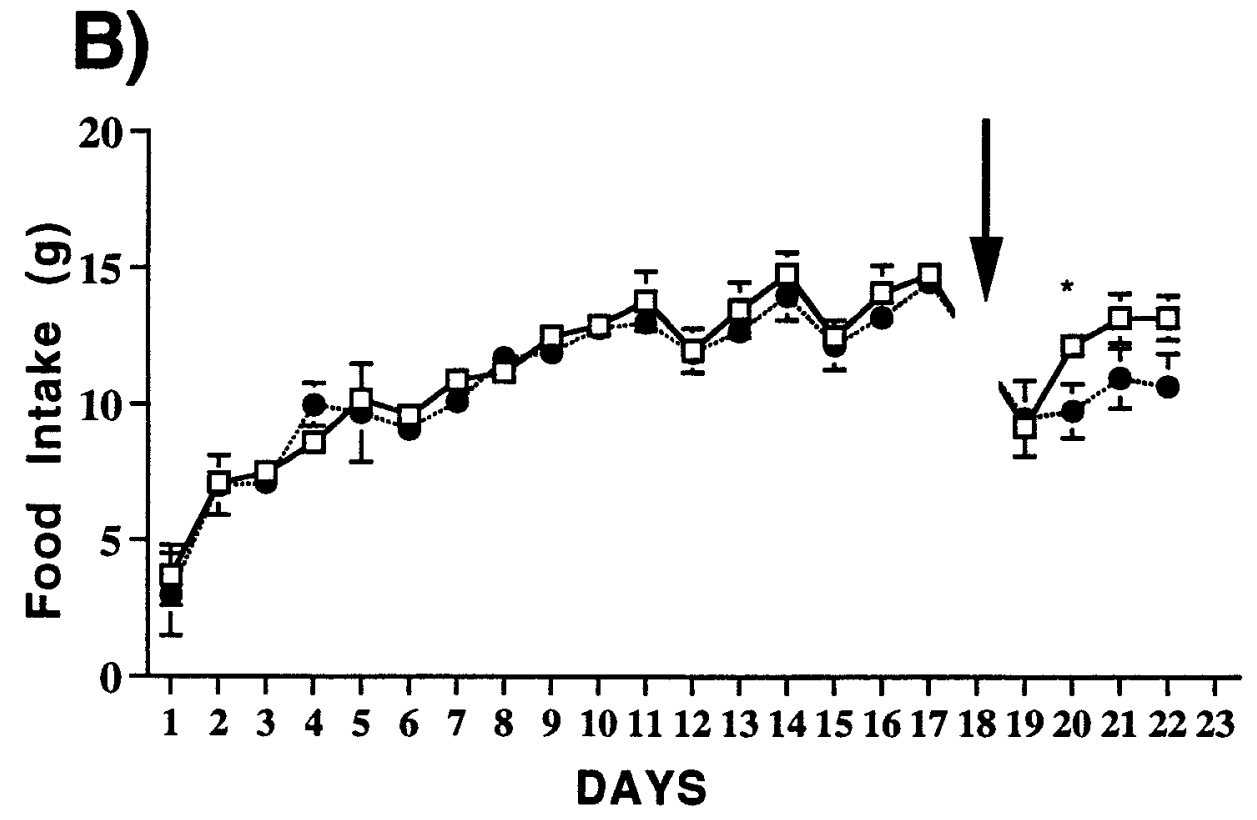

missed light and dark groups there were $120 \pm 26$ and $216 \pm 51$ c-Fos-stained cells, respectively. The small number of rats does not yield either a significant pattern in VMN (observed in control, RF rats) or a significant difference compared with RF rats with bilateral injections that show equal numbers of c-Fos cells in light and dark (Fig. 9). In these rats, c-Fos in the medial pvTHAL was $285 \pm 52$ and $368 \pm 29$ cells in the light and dark, respectively, again suggesting a light/dark pattern in c-Fos cell number, like that in control rats (Fig. 8). The number of cells stained with c-Fos in the anterior sPVNz in these rats was $206 \pm 35$ in the light and $292 \pm 39$ in the dark and in the posterior sPVNz was $145 \pm$ 34 in the light and $218 \pm 85$ in the dark. The lack of significant difference between light and dark counts in the sPVNz demonstrates that colchicine injections that miss the VMN do not cause the gain in neuronal activity pattern that is similar in phase to that of the SCN.

\section{DISCUSSION}

In rats fed ad libitum, presumed disruption of normal VMN activity by colchicine treatment diminishes the amplitude of rhythms mediated by other hypothalamic cell groups (e.g., DMN, locomotor activity; PVN, eating, drinking, and HPA activity; mPOA, body temperature; see introductory remarks) and blocks light/dark c-Fos patterns in all sites except for SCN and anterior and medial pvTHAL. Thus, the VMN appear normally to augment the circadian rhythms imposed on other brain structures by activity in the SCN. Rats fed ad libitum have c-Fos patterns in phase with their nocturnal activity cycle and inversely related to the light-entrained, endogenous SCN pattern. RF successfully entrains peak HPA activity to the time of feeding and induces a marked c-Fos pattern in the VMN. However, although it generally increases the numbers of c-Fos cells, RF does not affect the light/dark pattern of c-Fos in any other nuclei. In RF rats, disruption of VMN activity blocks patterns in both HPA activity and c-Fos in all hypothalamic and thalamic sites but not SCN, suggesting that it is the RF-induced c-Fos pattern in VMN that determines activity in these other cell groups. When feeding is reduced and out of phase with the light/dark cycle and the VMN are inactivated, significant patterns in c-Fos appear in the sPVNz 


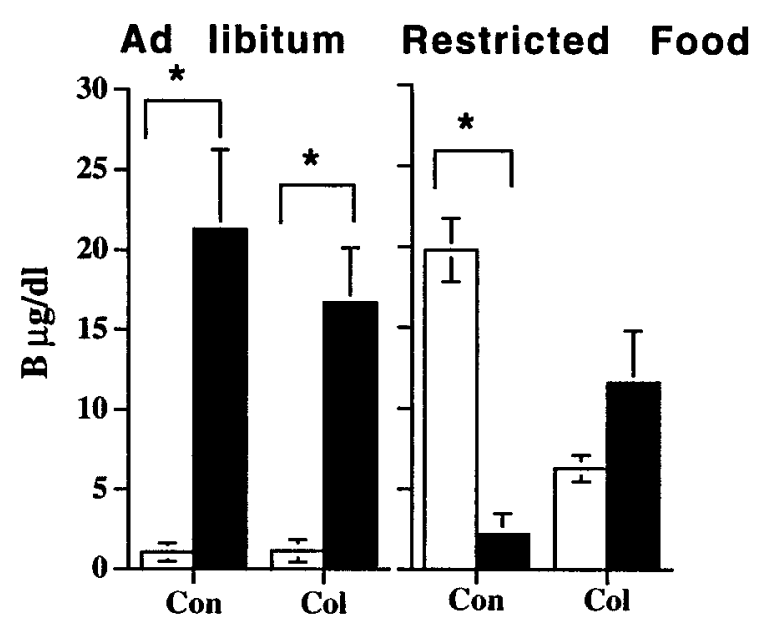

Figure 7. Basal plasma B levels before ad libitum feeding and after food restriction. Same rats as in Figure 6. After ad libitum feeding, there is a significant diurnal rhythm in basal $\mathrm{B}$, which peaks in the dark $\left({ }^{*} p<\right.$ 0.0001). After restricted food, saline-injected animals show a distinct reversal in the diurnal pattern of B levels $(p<0.0001)$. However, there was no rhythm in colchicine-injected animals. White bars, Early light (9:00 A.M.); black bars, late light (5:00 P.M.); Con, Saline-injected control; Col, colchicine-injected rat.

that uniquely parallel the SCN pattern, suggesting that cells in the sPVNz respond to inputs related to both energy balance and SCN output.

It is possible that colchicine injections affect not only cells in the VMN but also fibers of passage. Although we have no direct evidence that the effects of colchicine are exerted only on cells in the VMN, we suspect that this is the case. Our previous results demonstrated that colchicine disruption of VMN activity produced effects identical to those reported after damage of the VMN with neurotoxins that presumably destroy cell bodies but spare fibers of passage (Choi et al., 1996). Recent studies have shown that colchicine injected into the VMN produces sitespecific effects, different from those observed after injections into the ARC, PVN, and DMN (Choi et al., 1997). Thus, altered activity in the VMN seems essential to the results of these experiments.

\section{Ad libitum and restricted feeding: control rats}

In animals fed ad libitum, all rhythms peak at or during the dark phase, and c-Fos levels in hypothalamic and thalamic nuclei are higher in the dark (except for light = dark in sPVNz, VMN, and ARC). c-Fos expression in the light-entrained SCN was opposite: higher in light than dark. The lack of a pattern in the sPVNz was surprising, because this is a major target of the SCN (Watts et al., 1987); however, it is also strongly innervated by afferents from the VMN (Canteras et al., 1994) and visceral inputs (e.g., from the parabrachial nuclei) (Bester et al., 1997). Cells in the sPVNz may "listen" to at least these three inputs. Activity reflected by c-Fos in the VMN is low in both light and dark in rats fed ad libitum; thus the VMN may not normally contribute a light/dark bias to excitatory activity in the sPVNz.

RF in light shifts HPA activity so that it peaks before feeding. It is probable that the peaks in other rhythms measured in rats fed ad libitum were also shifted by RF, as shown by Krieger (1980) and others (Dallman, 1984; Mistlberger, 1994). In most nuclei, c-Fos cell numbers remain higher in dark, but the number of active cells was increased at both times, suggesting a generalized

\section{Control Colchicine}

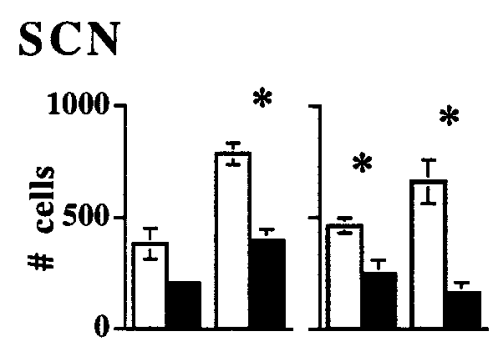

sPVNz-ant

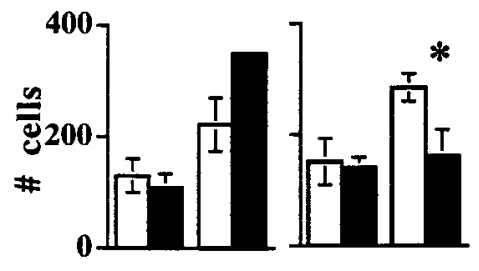

\section{sPVNz-post}

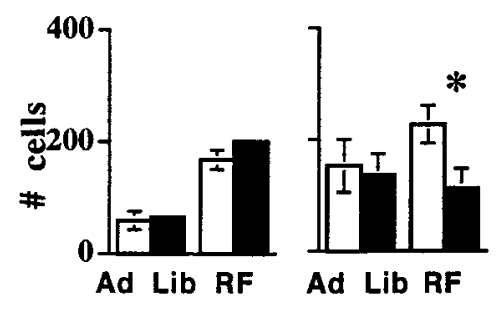

Figure 8. Under ad libitum ( $A d$ Lib) and restricted feeding $(R F)$ conditions, the number of c-Fos immunostained cells in the suprachiasmatic nuclei $(S C N)$, and anterior and posterior portions of the hypothalamic subparaventricular zone ( $s P V N z$-ant and $s P V N z$-post, respectively) are shown $5 \mathrm{~d}$ after colchicine or saline injections into the VMN. *Significance $(\mathrm{p} \leq 0.05)$ between numbers of cells in each structure from brains collected during the light (white bars) and dark (black bars). The number of rats measured at each site is at least five per group except for SCN in the rats fed ad libitum, in which cell numbers of four saline- and three colchicine-injected rats were measured in the light. Error bars indicate SEM. They are present, although some may be too small to be visible.

effect of decreased food intake and body weight gain. RF abolishes the pattern in the DMN with increased c-Fos in the light, possibly as a consequence of increased preprandial activity. Neuronal activity in the VMN develops a light $<$ dark pattern in $\mathrm{RF}$ and may be responsible for maintaining normal light $<$ dark $\mathrm{cFos}$ patterns elsewhere during RF. Although RF increases c-Fos expression in the $\mathrm{SCN}$, cell numbers remain light $>$ dark. c-Fos in the $\mathrm{sPVNz}$ remains without a light/dark difference when a marked c-Fos rhythm is exhibited in the VMN.

\section{Ad libitum and restricted feeding: VMN disruption}

In both ad libitum and RF animals, colchicine-induced disruption of the VMN diminishes rhythm amplitudes and abolishes light/ dark c-Fos patterns in all hypothalamic nuclei except the SCN. These results strongly support the notion that when normally active, the VMN regulate activity in the other nuclei and also determine the shift in corticosterone that occurs with RF. It is unlikely that shifts in SCN activity cause the shifted rhythms, 


\section{Control Colchicine}

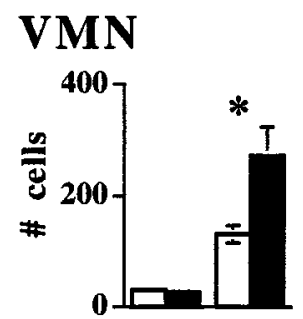

DMN

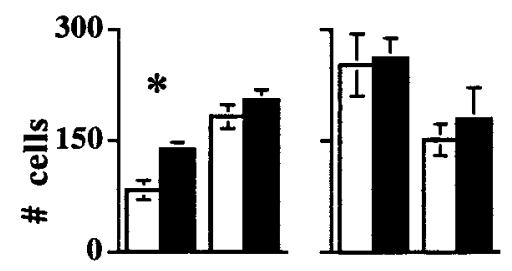

\section{pPVN}

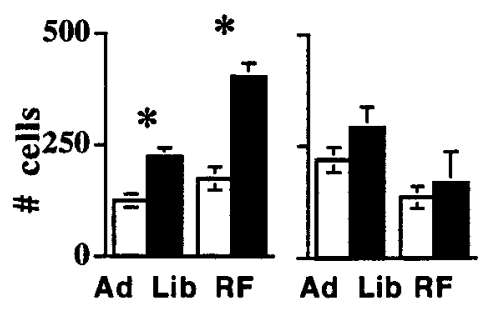

Figure 9. Under ad libitum (Ad Lib) and restricted feeding $(R F)$ conditions, the number of c-Fos-immunostained cells in the anterior VMN $(V M N)$, the dorsomedial nuclei $(D M N)$, and the parvocellular paraventricular nuclei $(p P V N) 5 \mathrm{~d}$ after saline and colchicine were injected into the VMN. *Significance $(p \leq 0.05)$ between numbers of cells in each structure from brains collected during the light (white bars) and dark (black bars). The number of rats per group is at least five.

because c-Fos patterns in the SCN were unaffected by both RF and colchicine injections into the VMN. VMN disruption blocked the c-Fos pattern in the posterior pvTHAL in rats fed ad libitum and in all three regions of the pvTHAL in RF rats. Although the VMN project to the entire pvTHAL, heavier projections to the posterior pvTHAL (Saper et al., 1976; Canteras et al., 1994) may override the large SCN input under conditions of ad libitum feeding. By contrast, when feeding and light are out of phase, inputs from the VMN and other cell groups to the pvTHAL appear to dominate.

In rats with $\mathrm{VMN}$ disruption fed ad libitum, there was no light/dark c-Fos pattern in the sPVNz. However, after RF and VMN disruption, a pattern in c-Fos appears in the sPVNz that is similar to the pattern in SCN. We suggest that this results from the removal of signals to the $\mathrm{SPVNz}$ of both dark phase inputs from food intake and increased dark phase activity that occurs in VMN under RF. When energy intake is reduced and "out of phase" with the light cycle, disruption of VMN function allows input from SCN to $\mathrm{PVNN}$ to dominate, thus inducing a unique pattern in the sPVNz that is out of phase with all other hypothalamic c-Fos patterns.

Two studies indicate that the sPVNz may be physiologically important. Lesions in the sPVNz reduced luteinizing hormone surges in proportion to the reduction of vasoactive intestinal

\section{Control Colchicine}

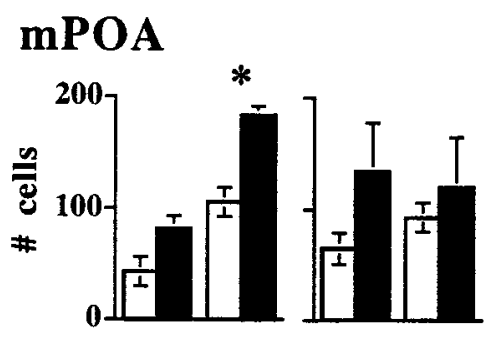

\section{mPVN}
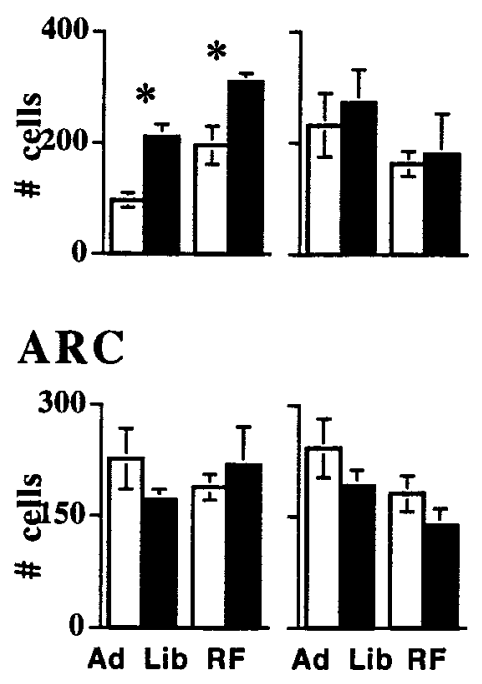

Figure 10. Under ad libitum (Ad Lib) and restricted feeding $(R F)$ conditions, the number of c-Fos-immunostained cells in the medial preoptic area $(m P O A)$, magnocellular paraventricular nuclei $(m P V N)$, and arcuate nuclei $(A R C) 5 \mathrm{~d}$ after colchicine or saline injections into the VMN. *Significance $(p \leq 0.05)$ between numbers of cells in each structure from brains collected during the light (white bars) and dark (black bars). The number of rats per group is at least five.

peptide-immunoreactive staining (from $\mathrm{SCN}$ ) in the sPVNz, suggesting that this region is critical for the SCN-induced, lightsensitive proestrous surge (Watts et al., 1989). Of VMN cells stimulated by systemic leptin to express c-Fos, $39 \%$ were also labeled with cholera toxin-b transported from the sPVNz (Elmquist et al., 1998), evidence that neurons in VMN stimulated by leptin send major projections to the sPVNz. Thus the sPVNz is clearly a target of, and could integrate information from, both the SCN and VMN.

The results strongly suggest that the VMN normally serve to amplify, in other hypothalamic nuclei, the oscillatory output from the circadian clock in the SCN. The VMN also are required to entrain rhythms to RF in the dynamic phase. RF alters VMN activity in control rats, inducing a marked c-Fos pattern similar in phase to the light/dark patterns that are maintained in other hypothalamic nuclei. VMN with colchicine blocks both patterns in HPA activity and neural (c-Fos) activity in other hypothalamic nuclei under both ad libitum and RF conditions. Is the VMN an important conduit for an endogenous food-associated oscillator, or is it an oscillator? Many studies have shown that lesions of the VMN block induction of food-entrained rhythms during the acute phase when obesity is occurring (for review, see Dallman, 1984; Mistlberger, 1994). However, two studies have shown that 


\section{Control Colchicine}
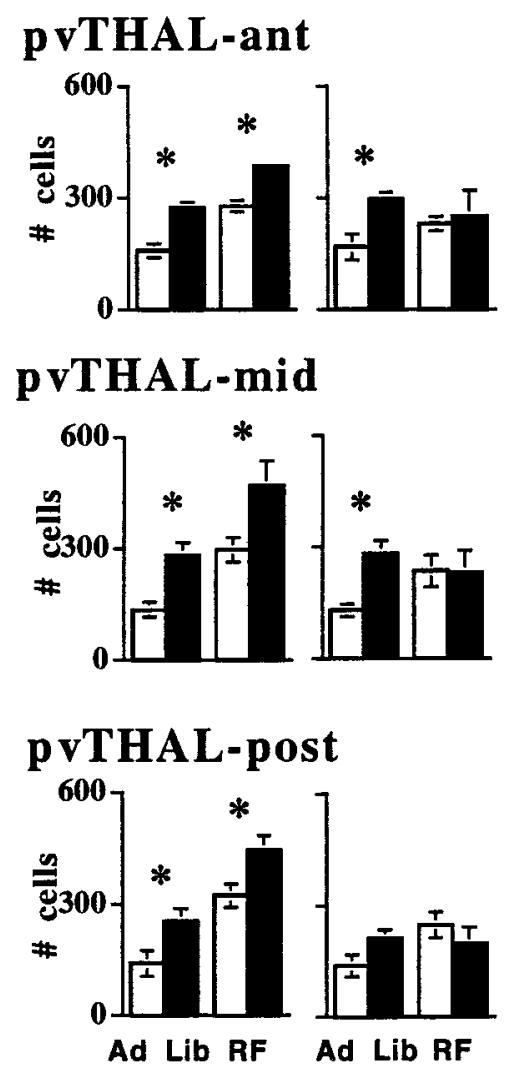

Figure 11. Under ad libitum (Ad Lib) and restricted feeding $(R F)$ conditions, the number of c-Fos immunostained cells in the anterior (ant), medial (mid), and posterior ( post) paraventricular thalamus ( $p v T H A L)$ $5 \mathrm{~d}$ after colchicine or saline injections into the VMN. *Significance $(p \leq$ 0.05 ) between numbers of cells in each structure from brains collected during the light (white bars) and dark (black bars). The number of rats per group is at least five.

activity and corticosterone rhythms can be entrained to RF in rats with VMN lesions during the static phase occurring months after lesions are made (Honma et al., 1984; Mistlberger and Rechtschaffen, 1984). These results suggest that the food-entrained oscillator is not in VMN. However, there is evidence for more than one food-entrained oscillator (Stephan and Becker, 1989), and either or both may lie outside the brain (Mistlberger, 1994). It is possible that the VMN serve as one of several food-entrained oscillators. The slow return of RF-induced rhythms in VMNlesioned rats during the static phase (Mistlberger and Rechtschaffen, 1984) could reflect an input from a second foodassociated oscillator that responds to the greater decrease of food intake during RF in VMN-lesioned rats, compared with controls (Honma et al., 1987). In this study, results from VMN-disrupted rats without acute phase obesity show that blockade of RF entrainment does not require preceding obesity and increased food intake.

We propose that the VMN are food-entrained oscillators. Neurons in VMN contain insulin and leptin receptors (Levin and Routh, 1996) and thus receive hormonal signals of small daily gains and losses in calorie storage. Leptin and insulin increase sharply after RF, whereas corticosterone falls (Ahima et al., 1998), providing strong, food-locked signals to the VMN. These may entrain a normally subservient VMN oscillator. Support for this notion requires demonstration that the RF-induced pattern in VMN c-Fos is sustained for at least one cycle after ending an $\mathrm{RF}$ regimen.

As pointed out by Mistlberger (1994), the utility of having endogenous food- in addition to light-entrained oscillators is considerable. When food is scarce, it may be available during a time of day usually associated with inactivity. Having foodentrained oscillators impose rhythms under these conditions would allow activity and other rhythms to peak at unusual times that could optimize locating, ingesting, and storing essential calories.

\section{REFERENCES}

Ahima RS, Prabakaran D, Flier JS (1998) Postnatal leptin surge and regulation of circadian rhythm of leptin by feeding: implications for energy homeostasis and neuroendocrine function. J Clin Invest 101:1020-1027.

Akana SF, Dallman MF, Bradbury MJ, Scribner KA, Strack AM, Walker C (1992) Feedback and facilitation in the adrenocortical system: unmasking facilitation by partial inhibition of the glucocorticoid response to prior stress. Endocrinology 131:57-68.

Bester H, Besson J, Bernard J (1997) Organization of efferent projections from the parabrachial area to the hypothalamus: a Phaseolus vulgaris-leucoagglutinin study in the rat. J Comp Neurol 383:245-281.

Bhatnagar S, Dallman MF (1998) Neuroanatomical basis for facilitation of hypothalamic-pituitary-adrenal responses to a novel stressor after chronic stress. Neuroscience, in press.

Bolles RC, Stokes LW (1965) Rat's anticipation of diurnal and a-diurnal feeding. J Comp Physiol Psychol 60:290-294.

Canteras NS, Simerly RB, Swanson LW (1994) Organization of projections from the ventromedial nucleus of the hypothalamus: a Phaseolusvulgaris-leucoagglutinin study in the rat. J Comp Neurol 348:41-79.

Choi S, Horsley C, Aguila S, Dallman MF (1996) The hypothalamic ventromedial nuclei couple activity in the hypothalamo-pituitaryadrenal axis to the morning fed or fasted state. J Neurosci 16:8170-8180.

Choi S, Hanson ES, Chu A, Horsley C, Dallman MF (1997) Inhibition of medial hypothalamic cell groups provides several roads to obesity in rats. Soc Neurosci Abstr 23:234.12.

Dallman MF (1984) Viewing the ventromedial hypothalamus from the adrenal gland. Am J Physiol 246:R1-R11.

Dallman MF, Strack AM, Akana SF, Bradbury MJ, Hanson ES, Scribner KA, Smith M (1993) Feast and Famine: critical role of glucocorticoids with insulin in daily energy flow. Front Neuroendocrinol 14:303-347.

Dragunow M, Faull R (1989) The use of c-fos as a metabolic marker in neuronal pathway tracing. J Neurosci Methods 29:261-265.

Elmquist JK, Ahima RS, Elias CF, Flier JS, Saper CB (1998) Leptin activates distinct projections from the dorsomedial and ventromedial hypothalamic nuclei. Proc Natl Acad Sci USA 95:741-746.

Gray GD, Bergfors AM, Levin R, Levine S (1978) Comparison of the effects of restricted morning or evening water intake on adrenocortical activity in female rats. Neuroendocrinology 25:236-246.

Green DJ, Gillette R (1982) Circadian rhythm of firing rate recorded from single cells in the rat suprachiasmatic brain slice. Brain Res 245:198-200.

Hanson ES, Dallman MF (1995) Neuropeptide Y (NPY) may integrate responses of hypothalamic feeding systems and the hypothalamopituitary-adrenal axis. J Neuroendocrinol 7:273-279.

Honma K, vonGoetz C, Aschoff J (1983a) Effects of restricted daily feeding on freerunning circadian rhythms in rats. Physiol Behav 30:905-913.

Honma K, Honma S, Hiroshige T (1983b) Critical role of food amount for prefeeding corticosterone peak in rats. Am J Physiol 245:R339-R344.

Honma K, Honma S, Hiroshige T (1984) Feeding-associated corticosterone peak in rats under various feeding cycles. Am J Physiol 246:R721-R726.

Honma S, Honma K, Nagasaka T, Hiroshige T (1987) The ventromedial hypothalamic nucleus is not essential for the prefeeding corticosterone peak in rats under restricted daily feeding. Physiol Behav 39:211-215.

Inouye ST (1983) Does the ventromedial hypothalamic nucleus contain a self-sustained circadian oscillator associated with periodic feedings? Brain Res 279:53-63. 
Inouye ST, Kawamura H (1979) Persistence of circadian rhythmicity in a mammalian hypothalamic "island" containing the suprachiasmatic nucleus. Proc Natl Acad Sci USA 76:5962-5966.

Johnson JT, Levine S (1973) Influence of water deprivation on adrenocortical rhythms. Neuroendocrinology 11:268-273.

Koibuchi N, Sakai M, Watanabe K, Yamaoka S (1992) Change in Foslike immunoreactivity in the suprachiasmatic nucleus in the adult male rat. NeuroReport 3:501-504.

Krieger DT (1974) Food and water restriction shifts corticosterone, temperature, activity and brain amine periodicity. Endocrinology 95:1195-1201.

Krieger DT (1980) Ventromedial hypothalamic lesions abolish foodshifted circadian adrenal and temperature rhythmicity. Endocrinology 106:649-654.

Krieger DT, Hauser H, Krey LC (1977) Suprachiasmatic nuclear lesions do not abolish food-shifted circadian adrenal and temperature rhythmicity. Science 197:398-399.

Levin BE, Routh VH (1996) Role of the brain in energy balance and obesity. Am J Physiol 271:R491-R500.

Lewis DE, Shellard L, Koeslag DG, Boer DE, McCarthy HD, McKibbin PE, Russell JC, Williams G (1993) Intense exercise and food restriction cause similar hypothalamic neuropeptide Y increases in rats. Am J Physiol 264:E279-E284.

Luiten PGM, Ter Horst GJ, Steffens AB (1987) The hypothalamus, intrinsic connections and outflow pathways to the endocrine system in relation to the control of feeding and metabolism. Prog Neurobiol 28:1-54.

Meijer JH, Schaap J, Watanabe K, Albus H (1997) Multiunit activity recordings in the suprachiasmatic nuclei: in vivo versus in vitro models. Brain Res 753:322-327.

Mistlberger RE (1994) Circadian food-anticipatory activity: formal models and physiological mechanisms. Neurosci Biobehav Rev 18:171-195.

Mistlberger RE, Rechtschaffen A (1984) Recovery of anticipatory activity to restricted feeding in rats with ventromedial hypothalamic lesions. Physiol Behav 33:227-235.

Moberg GP, Bellinger LL, Mendel VE (1975) Effect of meal feeding on daily rhythms of plasma corticosterone and growth hormone in the rat. Neuroendocrinology 19:160-169.

Moga MM, Weis RP, Moore RY (1995) Efferent projections of the paraventricular thalamic nucleus in the rat. J Comp Neurol 359:221-238
Mouret JR, Bobillier P (1971) Diurnal rhythms of sleep in the rat: augmentation of paradoxical sleep following alterations of the feeding schedule. Int J Neurosci 2:265-270.

Paxinos G, Watson C (1986) The rat brain in stereotaxic coordinates. London: Academic.

Peng Z, Grassi-Zucconi G, Bentivoglio M (1995) Fos-related protein expression in the midline paraventricular nucleus of the rat thalamus: basal oscillation and relationship with limbic efferents. Exp Brain Res 104:21-29.

Persons JE, Stephan FK, Bays ME (1993) Diet-induced obesity attenuates anticipation of food access in rats. Physiol Behav 54:55-64.

Refinetti R, Menaker M (1992) The circadian rhythm of body temperature. Physiol Behav 51:613-637.

Sagar SM, Sharp FR, Curran T (1988) Expression of c-fos protein in brain: metabolic mapping at the cellular level. Science 240:1328-1331.

Saper CB, Swanson LW, Cowan WM (1976) The efferent connections of the ventromedial nucleus of the hypothalamus of the rat. J Comp Neurol 169:409-442.

Stephan FK, Becker G (1989) Entrainment of anticipatory activity to various durations of food access. Physiol Behav 46:731-741.

Stephan FK, Zucker I (1972) Circadian rhythms in drinking behavior and locomotor activity of rats are eliminated by hypothalamic lesions. Proc Natl Acad Sci USA 69:1583-1586.

Tokunaga K, Fukushima M, Kemnitz JW, Bray GA (1986) Comparison of ventromedial and paraventricular lesions in rats that become obese. Am J Physiol 251:R1221-R1227.

Touzani K, Velley L (1992) Ibotenic acid lesion of the hypothalamic paraventricular nucleus produces weight gain but modifies neither preference nor aversion for saccharin. Physiol Behav 52:673-678.

Turek FW (1985) Circadian neural rhythms in mammals. Annu Rev Physiol 47:49-64.

Watts AG, Swanson LW, Sanchez-Watts G (1987) Efferent projections of the suprachiasmatic nucleus: I. Studies using anterograde transport of Phaseolus vulgaris leucoagglutinin in the rat. J Comp Neurol 258:204-229.

Watts AG, Sheward WJ, Whale D, Fink G (1989) The effects of knife cuts in the sub-paraventricular zone of the female rat hypothalamus on oestrogen-induced diurnal surges of plasma prolactin and $\mathrm{LH}$, and circadian wheel-running activity. J Endocrinol 122:593-604.

Wilkinson CW, Shinsako J, Dallman MF (1979) Daily rhythms in adrenal responsiveness to adrenocorticotropin are determined primarily by the time of feeding in the rat. Endocrinology 104:350-358. 